\title{
Safety Behavior Checklist for Scrubber Installation Activities at Construction Site
}

\author{
Khidir Sanusi, Syuhaida Ismail and Normawati Mohd Shariff \\ UTM RAZAK School of Engineering and Advanced Technology, \\ Universiti Teknologi Malaysia Kuala Lumpur, Malaysia
}

Correspondence should be addressed to: Khidir Sanusi; khidir.plc@gmail.com

Received date: 24 August 2015; Accepted date: 25 October 2016; Published date: 31 May 2017

Academic Editor: Norhaya Kamarudin

Copyright (C) 2017. Khidir Sanusi, Syuhaida Ismail and Normawati Mohd Shariff . Distributed under Creative Commons CC-BY 4.0

\begin{abstract}
Rapid development and technological progress within the nation have significantly affected the performance of construction industry in Malaysia. Likewise, the risk of accidents at construction sites is increasing correspondingly as much of construction work involves the wide-use of machinery, equipment and technologies. Moreover, construction workers are still lacking awareness of safety culture and safety tools application. Safety professionals have suggested that most work-related accidents result from employees committing unsafe acts rather than unsafe conditions. Organization X, a Malaysian project-based company which specializes in the installation of scrubber systems that control the generation of pollution into the atmosphere was chosen as the case study in investigating the at-risk behaviors that construction workers may pose and perform during the installation activities of scrubbers. Scrubber installation was selected as in year 2013 alone, 39 cases of incidents were recorded when installing scrubbers at construction sites. The prevalence of such incidents not only presented an economic burden due to increased medical expenses and claims, but has also affected the work efficiency, resulting in deterioration of the company's performance and reputation. Hence, this study investigated the use of behavior-based safety (BBS) approach to control and mitigate accidents during at-site scrubber installation. A customized Safety Behavior Checklist (SBC) was employed to minimize unsafe acts and behaviors committed by workers. It also studied the impact of implementing the SBC and applying intervention initiatives on observed at-risk behaviors of workers. It is found that the implementation of customized SBC contributes to modify and subsequently improve workers' safe behavior.
\end{abstract}

Keywords: Customized Safety Behavior Checklist, Scrubber Installation, Construction

Cite this Article as: Khidir Sanusi, Syuhaida Ismail and Normawati Mohd Shariff (2017)," Safety Behavior Checklist for Scrubber Installation Activities at Construction Site", Journal of Southeast Asian Research, Vol. 2017 (2017), Article ID 870456, DOI: 10.5171/2017.870456 


\section{Introduction}

The challenge to improve safety at work remains a key concern to many organizations, especially as traditional approaches to safety in organizations have not demonstrated the anticipated impact of reducing accident rates. Historically, organizations have focused on improving safety by addressing the work environment surrounding employees, providing hazardfree facilities, providing better tools and equipment without achieving any appreciable reduction in the rate of accidents. Organizations that are focused on outcomes and result-oriented often unintentionally encourage work culture that overlooks or disregards at-risk behaviors (Gilmore and Perdue, 2001). However, no matter how safely a workplace is designed, or how thoroughly employees are trained, or how stringently safety compliance is enforced, organizations must still deal with the uncertainty of human behavior. People are not perfect and will make mistakes despite their best intentions and working in the best surroundings.

Thus, behavior-based safety has become a popular way of managing the people side of safety since it revolves around what motivates and reinforces people's behavior. According to behavioral science research, the three most significant factors that determine a worker's behavior are organizational structure, individual attitudes, and the consequences of the behavior (Krause, 1991). Organizational culture has an important impact on safety. It can unintentionally encourage unsafe behavior or foster beliefs that are opposed to safety. People who work in an organization become aware of its values and climate from observing how coworkers behave and how things are done. This awareness affects the worker's behavior. Krause (1991) further claimed that attitude, the positive and negative feelings and thoughts that affect outlook and perception of the environment, is the second factor that can affect an individual's behavior. However, the relationship between attitude and behavior is not straightforward, as consequences control behavior (Krause, 1991). It is key to understanding why people act the way they do. It also explains the special challenges of promoting safety and health in the workplace.

Based on Skinner's operant theory, behavior is motivated by events or conditions that follow it (Skinner, 1953). Pleasant consequences increase behavior and unpleasant consequences decrease behavior. At-risk behaviors and unsafe acts are often followed by feelings of excitement, comfort and convenience, while safe and healthy behaviors are often accompanied by inconvenience, discomfort, or even boredom. In addition, the presumed benefits of safe and healthy behaviors are either not experienced or delayed. The promotion of this theory within the behavioral safety field (Geller, 2005; McSween, 2002; Krause, 1997) has led many to believe that the antecedentbehavior-consequence (ABC) model focuses almost exclusively on the psychology of safety especially in the construction industry.

The focus of Behavior Based Safety (BBS) approach is on identifying the specific consequences that control essential or critical safety-related behaviors. Consequences are the most powerful motivators that determine behavior. Thus, to change unsafe behavior, there is a need to identify what consequences are motivating workers. The basic tools of BBS can be applied to improve the behaviors of every employee in an organization, from the worker performing the hands-on activities to the supervisor overseeing the entire effort. The approach consists of a wide range of tools, such as checklists, inspections, safety samplings, benchmarking, attitude surveys, documents and record analysis as well as involvement of experts and consultants.

\section{Critical Behavior Checklist (CBC)}


The critical behavior checklist (CBC) is believed to be one of the most valuable Behavior Based Safety (BBS) tools. Generally a CBC consists of a list of some of the specific behaviors required to complete a task effectively. The descriptions and examples in CBC address safety, and it is vital for improving competence at any task. Performance cannot improve without behavior-based feedback, and feedback is often most informative when linked to observations recorded on a CBC (Geller, 2000). Haupt (2001) developed the Safety Behavior Checklist (SBC), a unique approach, based on the New Zealand regulations (Occupational Safety and Health Service, 1995). This is based on the assumption that since accidents are more likely to have originated from unsafe acts and at-risk behaviors of employees, the identification of these behaviors can be made using an SBC. The potential of accidents can be thus alleviated by improving workers' safe behavior and eliminating their at-risk behaviors. Williams (2000) and Wayne (2004) identified and employed different approaches on implementing safety observation checklist. Both researchers used generic observation checklists and safety observation surveys respectively to improve the respondents' safety performance.

However, focusing on peoples' behavior remains a subject of debate among safety professionals. Critics claim that BBS put the blame of safety failures on workers, without taking into account the whole system. Many argued that using generic observation checklists meant that observations are not conducted on specific activities. Additionally, they did not account for unsafe acts or at-risk behaviors that workers are likely to do when performing certain activities. Likewise, results from safety observation surveys are uncertain due to the respondents' tendency to fill the survey forms recklessly (Cornish, 2002). Manuele (2002) claimed that basing safety efforts on the premise that man failure causes most accidents, the preventive efforts are directed at the worker rather than on the operating system in which the work is done. Despite criticisms that BBS tends to emphasize behavior, BBS should be recognized for focusing on the human side of safety. It is believed that it can be a powerful tool for achieving continuous improvement in safety performance.

\section{The Study}

This paper is based on the premise of Behavior Based Safety (BBS), an approach that focuses on identifying and changing unsafe worker behaviors. Since accidents generally originate from the at-risk behaviors of people, they can be prevented through the identification and elimination of these behaviors via a customized Safety Behavior Checklist (SBC). Hence, the study was undertaken with the objective of investigating the at-risk behaviors that construction workers may pose and perform during installation activities of scrubber through observing behaviors of workers involved during at-site installation of scrubbers. It also intended to examine the impact of implementing the SBC and observed behaviors of workers involved during at-site installation of scrubbers. The motivation to the initiative is to establish whether construction workers' safe culture can be improved via behavior modification, reducing the potential of accident events at the work site.

Organization $\mathrm{X}$ was selected as the case study of this investigation. Between 2011 and 2013, Organization X had engaged in more than 25 projects that involved in the installation of scrubber systems. A scrubber is an engineering control system that functions as a form of filtration system that controls the generation of air pollutants before release into the atmosphere. The installation of such a system is a high-risk activity involving working at height, using heavy lifting machinery and equipment as well as in hot work conditions. Based on the Organization X's safety record from January 2013 until December 2013, a total of 36 incidents have been recorded. 19 cases of near misses, 12 cases of employee injury, and 5 cases of property damage were reported in the process of installing scrubbers. Even though no fatality was 
recorded, all these incidents fell into the fret category, which meant that they have the potential of affecting workers' safety as well as organization's reputation.

The process of installation of a scrubber involves several stages, starting from its arrival, to unloading, to secure installation and mounting at the designated location (AAF International, 2014; Hensen, 2012 and MAPCO, 2006). In order to unload the scrubber from delivery truck, the scrubber needs to be positioned in upright position, and then by using a crane or lifting machine, the scrubber will be lifted and shifted to a designated location (Harrington, 2005). Once it is in the exact desired position and location, workers will then conduct minor adjustments before mounting the bottom part of the scrubber column. At this point, workers are likely to be working at height. Throughout the process, the use of machineries and power tools are involved, and workers are required to wear personal protective equipment (PPE). Thus, during installation, several activities, which are categorized as high-risk tasks are performed. Workers tend to conduct these tasks without paying much heed to at-risk behavior, such as using bare hands to receive and adjust the lifted scrubber and improper use of tools, for example using a spanner for hammering. In order to achieve better safety performance throughout the installation process, project engineers, site supervisors and safety personnel must realize that their workers' safety is a priority for every installation made.

\section{Methodology}

This paper is based on a study using one of the Behavior Based Safety (BBS) tools, which is Safety Behavior Checklist (SBC) for a specific job and activity for scrubber installation at site. The study attempted to determine the actual nature of at-risk behavior and unsafe acts committed by construction workers of Organization $\mathrm{X}$ during installation of scrubbers. From the pre-study observations and discussions with safety expert at sites, a draft checklist was drafted. Individual tasks were analyzed and classified into five task- categories: personal protective equipment (PPE), housekeeping, tools and equipment use, communication and body positioning and protection into a Task Breakdown Form. A pilot test was conducted on independent observers who were then in charge for a scrubber installation project in Malaysia Northern region. The observers comprised of Organization $\mathrm{X}$ personnel from different backgrounds and roles in the organization. The purpose of selecting different ranges of background of observers is to test the usability of the checklist whether it can be easily adapted by all ranges of people from different backgrounds in Organization $\mathrm{X}$. Once feedbacks were gathered, a final checklist was developed and renamed the Observation Card (OBS).

During the actual observation phase, 45 respondents have been tested for the period of three months starting from January 2015 until March 2015. The observers for this phase comprised of Site Supervisors, Safety and Health Officers (SHO), Quality Assurance (QA) executives as well as Project Engineers. The selection of the stated observers is based on their work nature as they will be the frequent users of the customized Safety Behavior Checklist (SBC) and will work closely with the respondents for every project that involves installation of scrubber. These observers were briefed on their roles and responsibilities as well as goals to achieve.

\section{Results}

The customized Safety Behavior Checklist (SBC) monitored three main activities (i.e. safe access, excavations and roof work) in which risky activities were performed at the organization under observation. For every activity, statements that required the observer to determine whether the workers performed their tasks safely or otherwise were presented. The total amounts of at-risk behavior and safe behavior observed over three months were then sorted out in a Microsoft Excel worksheet and presented.

As per result obtained from the observation, the at-risk behaviors and safe 
behaviors of construction workers during scrubber installation were monitored. It was identified that most of the at-risk behaviors that fell in the danger (red) and critical (orange) levels were from the noncompliance with the personal protective equipment (PPE) requirement and improper body positioning and protection, as well as housekeeping and communication issues. Examples of PPE non-compliance were not wearing gloves or safety goggle or harness, and taking off helmets while climbing ladder. Improper Body Positioning and Protection included improper lifting posture, walking under suspended load, and using hands to position suspended scrubbers. At-risk housekeeping activities included obstructing exit doorways with tool boxes, not clearing construction debris and other trash; while at-risk communication activities included workers not intervening when their co-workers commit mistakes, not using the walkie-talkie to give instructions, and using ambiguous hand signals while communicating with crane operators. However, in terms of severity, most of at-risk behaviors were due to improper body position and protection.

The result from the first month of observation showed dismal outcomes for almost all criteria as the implementation of the customized SBC observation was new for most of the construction workers. A Safe Range Target (SRT) is the desired target for safe behavior. Based on the actual number of safe acts, it was determined whether the SRT for each criterion is within the PASS or FAIL range. Initially, the workers continued to perform many at-risk behaviors that they routinely conducted previously. The ratio of PASS to FAIL safe target range during the first week data was recorded at $1: 1$, which indicated that the workers committed as many unsafe acts as they did safe acts. Gradual improvements in subsequent weeks were noted, and the number of dangerous and critical level behaviors decreased. Overall, the PASS or FAIL safe range target recorded a higher percentage of PASS target with a ratio of 9:6 for PASS: FAIL safe target range. The very critical unsafe behavior observed for the first and second weeks was housekeeping. However, on the following third week, there was a discernable improvement of this criterion whereby the average (yellow) and safe (green) levels were achieved on the first and second days of the third week respectively.

By the second month, it can be seen that the proportion of PASS for safe range target was higher than FAIL recorded. This to some extent indicated a good sign of improvements of construction workers behaviors.

The improvements were achieved due to the process of developing a safe behavior. Interventions were done in order to make the workers aware and understand their roles in maintaining the safe workplace. In general, a Toolbox Meeting was conducted before workers commenced their work. During the meeting, the Safety and Health Officer (SHO) emphasized the safety precautions that needed to be taken while performing tasks, taking into consideration the SBC observations. The reminders were meant to ensure that workers behave safely, thus improving their safety performance. Authorizing and requiring workers to look after each other and intervene if their co-workers commit safety oversights when working as a team further improved results. It required time and intervention to make them realize that what they were doing was wrong and unsafe and subsequently modify their behavior. To educate them about safety while performing the job, the observers who conducted the SBC observation had to intervene and explain the right and the safe ways to do the job.

The result for the final month showed the greatest improvement if compared with the first two months of observation. Even though on the final month there is no prereminder during Toolbox Meetings by SHOs on the SBC observation, the result was encouraging. The general improvement was seen when the safe range target was successfully upgraded to the next level. Based on the outcomes of the result, all criteria observed were performed at either the average or safe 
levels. This somehow led to the improvement of the proportion of safe acts by workers at site, including workers' behavior towards their personal safety as well as safety of others. The improvement can be attributed to interventions of SHOs in emphasizing team safety performance.

\section{Conclusion}

This paper has shown that Behavior-Based Safety (BBS) has a place in achieving continuous improvement in safety performance. Although the biggest criticism against it is that it is an approach to safety that focuses solely on workers' behavior as the cause of most work-related injuries, it cannot be disputed that interfaced with sound engineering and administrative control, the approach can produce exceptional results. It is an initiative to employee driven safety.

The use of the customized Safety Behavior Checklist (SBC), a basic tool of BBS, can be applied to identify and subsequently modify construction workers' safety behavior, thus improving their safety performance, and indirectly enabling them to reduce the potential risks of accidents as well as save unnecessary costs caused by the accidents. This paper supports Geller (2000) who claimed that correct implementation of SBC can effectively contribute to risk management and injury prevention. It emphasizes that developing a practical behavioral checklist can help match at-risk behavior with the appropriate intervention initiatives that can not only correct and replace undesired behavior, but also encourage the internalization of desirable ones by empowering employees to take control of their own safety performance, thus reducing occupational risks and preventing workplace injuries.

\section{Acknowledgment}

The authors would like to express their sincere gratitude to the Ministry of Education Malaysia, Universiti Teknologi Malaysia (UTM) and the Research
Management Centre (RMC) of UTM for providing the financial support for this paper to be published. This study is financed by the Grant for Research University (GUP) of UTM for research funding under Cost Centre No. 11H09 and $11 \mathrm{~J} 75$.

\section{References}

1. AAF International (2014), SAAFTM PORTA-Scrubber, Installation, Operation and Maintenance Instructions, AAF International, Louisville, Kentucky

2. Bureau Veritas (2015), Exhaust Scrubbers what you need to know, Bureau Veritas, Marine and Offshore Division

3. Cooper, C.D. and Alley, F.C. (1994), Air Pollution Control: A Design Approach, 2nd ed. Waveland Press, Prospect Heights, Illinois

4. Cooper, M.D. (2003), Behavior-based safety stills a viable strategy. Safety and Health, 46(1):410-18

5. Cooper, M.D. (2008), “User survey report. 2008'. Franklin, IN: BehavioralSafety.com. [Online], [Retrieved April 15, 2014], http://www.behavioralsafety.com/survey results/finalsurveybscom1.html

6. Cornish, J. (2002), 'Response Problems in Surveys - Improving response \& minimizing the load', UNSD Regional Seminar on 'Good Practices in the Organization and Management of Statistical Systems' for ASEAN countries, Yangon Myanmar

7. EPA (1982), Control Techniques for Particulate Emissions from Stationary Sources, Volume 1 (EPA- 450/3-81-005a, NTIS PB83-127498), U.S. Environmental Protection Agency, Office of Air Quality Planning and Standards

8. Geller, E.S. (2005), 'Behavior-Based Safety and Occupational Risk Management', Behavior Modification, 29 (3): 539-561 
9. Gilmore, M.R., Perdue, S.R. and Wu, P. (2001), 'Behavior-Based Safety: The Next Step in Injury Prevention', SPE International Conference on Health, Safety \& Environment in Oil and Gas Exploration and Production, Kuala Lumpur, Malaysia, Society of Petroleum Engineers Inc.

10.Harrington (2005), Fiberglass Fume Scrubber Installation and Maintenance Instructions, Harrington Industrial Inc, California

11.Haupt, T.C. (2001), The Performance Approach to Construction Worker Safety and Health, University of Florida

12.Hensen, J.P. (2012), Exhaust Gas Scrubber Installed Onboard MV Ficaria Seaways, Mijostryrelsen, Danish Ministry of the Environment, Environmental Protection Agency

13. Howe, J. (1998), A union critique of behavior safety, ASSE Behavioral Safety Symposium, Orlando, FL

14.Jaegar (2007), Scrubbing Pollutants from Vent Streams, Waste Gas Scrubbing, Jaegar Products Inc, Houston, Texas

15.Krause, T.R. (1997), The behavior-based safety process, 2nd edition. New York: Van Nostrand Reinhold
16.Manuele, F.A. (2002), Heinrich Revisited: Truisms or Myths, 2nd edition. Itasca, IL: National Safety Council

17.MAPCO (2006), Installation Operation Maintenance Wet Scrubbers MW-100, MW200, MW-300, Midwest Air Products Co. Inc, Traverse City

18.McSween, T.E. (2002), The values-based safety process: Improving your safety culture with a

19.behavioral approach, 2nd edition. New York: John Wiley and Sons

20.Naso, M. (2002), Change in behavior: The decline of behavior-based safety gives way to safety management initiatives. Safety and Health, 5(1):33-39.

21.Skinner, B.F. (1953), Science and human behavior. New York: Macmillan

22.TexaSafe (2004), TexaSafe: A Guide to Total Safety Culture, Behavioral Based Safety-04 04

23.Wayne, D. (2004), An Analysis of the Behavior-Based Safety Program at Company XYZ, Research paper, The Graduate School, University of WisconsinStout

24.Williams, J.H. (2000), Using Behavioral Safety to Improve Safety Culture, Safety Performance Solutions, Blacksburg, VA 